\title{
Remediation of Water from Waste Lubricating Oil Spill Using Potato Peels
}

\author{
Paitoon Tontiwachwuthikul, Isam Al Zubaidi*, Eva Rennie, Spencer Schubert, Magda Seitz, Cassandra \\ Selinger-Silva \\ Faculty of Engineering and Applied Science, University of Regina, \\ SK, S4S 0A2, Canada \\ Isam.Al.Zubaidi@uregina.ca
}

\begin{abstract}
Potato peels was used for remediation of water from waste oil spill. The remediation process was investigated at room temperature, static state, and short and medium term adsorption process. The bouncy test, sorption capacity, retention time, and reusability were investigated. The adsorption equilibrium model and the kinetic model were determined. The potato peels was treated and passed the bouncy test. The oil sorption capacity was $2.15 \mathrm{~g}$ of oil/g sorbent. The reusability was not good as other natural sorbents. The adsorption process of using potato peels in adsorbing waste lubricating oil from the surface of water followed the second order kinetics adsorption process.
\end{abstract}

Keywords: adsorption process, waste lubricating oil, potato peels, water remediation.

\section{Introduction}

Oil spills cause many problems related to the oil countries as well as to all oil and transportation companies. Oil spill have an impact effect on marine life, quality of drinking water, tourism, economics, and environment. As an instance, BP Deep Horizon rig oil spill in 2010 was leading to the death of 11 workers and released approximately 5 million barrels of oil into the Gulf of Mexico. This oil disaster lasted for three months and leads to the death of a huge population of marine animals and polluted $320 \mathrm{~km}$ of shorelines. The water remediation process takes an intensive attention from different authors. An efficient and economical way of water remediation needs s to be investigated. One of these ways is the adsorption process using cheap and efficient materials. Sorbents are either natural or synthetic. This work will focus on the waste biomaterials such as potato peels.

Moss, straw, wool, sawdust, and peat are the natural sorbents were evaluated for oil spill [1]. Peat is one of the best materials to adsorb diesel oil from the surface of water. Kapok (Ceiba pentandra) fibre, an agricultural product, was evaluated as untreated and solvent-treated fibbers [2]. The kapok fibres, both at its natural state and after solvent treatments, demonstrated excellent oil /water separation. Rice husk was alkali treated at low temperatures aiming to produce a lingo cellulosic sorbent material and removing their silica [3]. Different treatment conditions of $\mathrm{NaOH}$ concentration, duration, and temperature were investigated. The optimized cellulosic sorbents show high marine fuel up take due to low bulk density and their fluffy structure. It is argued that the bulk density is a critical factor in the excellent uptake capacity. Other advantages include higher yields and lower treatment temperatures compared to other studies. The proposed cheap and environmentally friendly method provides a simple and safe technique for the production of valuable products from ligno-cellulosic agricultural waste. Luffa, an agricultural waste, was used as a sorbent material to study the adsorption capacity for different types of oil and water pickup [4]. The efficiency of fibres to remove crude oil from seawater was related to the surface properties of the fibres, concentration of the oil, amount of the fibres, as well as the temperature of the crude oil. This sorbent exhibited a good reusability since the decrease in sorption efficiency did not exceed $50 \%$ of the initial value after three sorption cycles. The effect of packing density, type of oils, and solvent treatment was studied in a batch system [5]. Oil sorption capacity, retention capacity, entrapment stability and reusability were evaluated. Kapok remained stable after fifteen cycles of reuse with only $30 \%$ of sorption capacity reduction. Root, stem and leaf of the dry biomass water hyacinth were studied [6]. Hydrophobicity, wettability (capillarity), buoyancy and sorption capacity of oils in the presence/ absence of water were evaluated. The oil sorption capacity increases with the increase of oil film thickness. The stem has a greater sorption capacity for diesel, lubricant, and castor oils. Arabic herbs Massofa and Youda were used as biodegradable organic 
natural sorbents [7]. The average oil sorption capacity was 6.281 and $5.415 \mathrm{~g}$ oil/g of sorbents for Youda and Masoofa. The effect of activation process of date palm kernel powder was investigated for remediation of oil-polluted water [8]. Date palm kernels were activated; it was found that the activation method of date palm kernel powder had a significant effect on oil sorption capacity. Spent malted barley was used for diesel oil adsorption from water [9]. The sorption capacity was $3.5 \mathrm{~g}$ of oil/g oil; the sorbent was able to keep about $75 \%$ of the adsorbed oil after three minutes of drainage. The sorption process followed the second order kinetics and Freundlich adsorption equilibrium isotherm model. Barley straw was used as oil sorbent material. The raw straw was pyrolyzed at $200-500{ }^{\circ} \mathrm{C}$ [10]. Oil sorption is dependent on the type of oil and the type of sorbent treatment. The carbonized barley straw showed good sorption properties and adequate reusability, indicating that a material based on natural fibres could be a variable alternative to commercially available synthetic materials that have poor biodegradability. Potato peels was used as solid bio waste material in removal of Ni (II) and dye removal from water using a batch process [11-12]. Potato ash was used to remove $\mathrm{Co}+2$ and $\mathrm{Eu}+3$ ions from aqueous solution. Characterization of the studied material was done using fourier transform infrared (FTIR), X-ray diffraction (XRD), surface area, thermogravimetric analysis (TGA) and differential thermal analysis (DTA). Experimental studies were conducted to evaluate and optimize the various process variables e.g. shaking time, $\mathrm{pH}$, initial ion concentration and the effect of interfering ions [13]. Potato peels are a waste biomaterial from potato during cooking process and especially from fast food restaurants. As an example, the 4500 Pounds of potatoes are used in Five Guys restaurants in Regina/ Canada /week. If the potato peels represent 10\% of the potato, then the amount of potato peels from one restaurant is $450 \mathrm{lbs}(250 \mathrm{Kg})$. There are two branches of this restaurant in Regina. So this restaurant generate $500 \mathrm{Kg} /$ week potato peels [14]. The water content of the potato peels is about $79 \%$, the potato peels powder will be $105 \mathrm{Kg}$ per week. The rrecovery, analysis, and utilization of potato peels were studied comprehensively by Narathip Yodsanti [15].

\section{Materials and Experimental Work}

\subsection{Materials}

Potato peals as solid bio waste material was used in this study due to the large quantity that generated in all houses and restaurant to prepare French Fries and especially in the fast food restaurants. Potato peals was washed with water several times to remove any dust or dirt material. Then it dried in an oven at $70{ }^{\circ} \mathrm{C}$ for one day then washed again and dried for another two days at $80^{\circ} \mathrm{C}$ to be sure that it is completely dried. The potato peals was crushed using mortar and pistol.

Waste lubricating oil from oil change carriage in Regina/ Canada was used in the contamination process of artificial seawater (3\% salt). The physical properties of the waste lubricating oil (Fuel) were measured according to ASTM standard methods as shown in the following table.

Table 1: Main physical properties of waste lubricating oil.

\begin{tabular}{|l|l|l|}
\hline Physical Properties & ASTM No. & Value \\
\hline Specific gravity & ASTM D 1298 & 0.851 \\
\hline API gravity & ASTM D 1298 & 34.77 \\
\hline Viscosity@ $40^{\circ}$ C, cSt. & ASTM D 445 & 55.060 \\
\hline Carbon residue, Conradson, wt\% & ASTM D 189 & 1.411 \\
\hline Ash content, wt, $\%$ & ASTM D 582 & 1.489 \\
\hline Sulphated ash, wt\% & ASTM D 874 & 1.21 \\
\hline Asphaltene content, heptane insoluble, wt\% & ASTM D 3279 & 0.802 \\
\hline
\end{tabular}

\subsection{Experimental work}

The experimental work was objected to measure the oil sorption capacity of potato peals, effect of quantity of sorbent, contact time with quantity of adsorbed oil, retention time, and reusability, packed column adsorption. Here are the main experimental steps to achieve these objectives. In order to evaluate the sorbancy of waste lubricating oil by potato peels, 5 samples of 1 gram through to 5 grams of waste lubricating oil was placed in a mixture containing $400 \mathrm{ml}$ of artificial seawater $(3.0 \% \mathrm{NaCl})$, and $40 \mathrm{~g}$ of waste fuel. The sorbent was left in the mixture for a total of 15 minutes, then extended for $1 \mathrm{hr}$ to be sure that the adsorption process is reached to equilibrium state. The strainer was removed and allowed to drain for 30 seconds before its weight was obtained. Using the pre-weight and post weight of the sample the amount of liquid that was 
adsorbed was determined. The water was then removed by rinsing the sorbent with n-heptane and then squeezing it to remove oil, water, and solvent. The amount of water was then determined. The amount of oil sorbed (oil sorption capacity $\mathrm{C}$, in $\mathrm{g}$ oil $\mathrm{g}^{-1}$ of sorbent mass) can be calculated according to:

$$
\mathrm{C}=\frac{m f-(m o+m w)}{m o}
$$

Where $\mathrm{m} f$ is the mass of the wet oil material after draining $(\mathrm{g})$, mo is the initial mass of dry material $(\mathrm{g})$ and $\mathrm{m} w$ is the water content in the material $(\mathrm{g})$.

The water uptake $\left(\mathrm{C}_{\mathrm{H} 2 \mathrm{O}}\right.$, in $\left.\mathrm{gg}^{-1}\right)$ after 30 seconds of drainage can be determined:

$$
\mathrm{C}_{\mathrm{H} 2 \mathrm{O}=\frac{m w t-m o}{m o}}
$$

Where $\mathrm{m}_{\mathrm{wt}}$ is the mass of the wet material after 30 seconds of drainage $(\mathrm{g})$, and $\mathrm{m}_{\mathrm{o}}$ is the initial mass of the material (g). The oil retention of the potato peels was determined by placing 1 gram of the sorbent in $150 \mathrm{ml}$ of waste fuel for 15 minutes. After the 15 minutes, the weights of the wet sorbent samples were taken at 10, 30, 60, 90, and 120 seconds. The quantity of oil retention was taken to be the difference between the dry sample and the weight of the wet sample - post drainage. The reusability of the potato peels was determined by placing a 1-gram sample of the sorbent in $150 \mathrm{ml}$ of waste fuel. The sorbent remained in the waste fuel for 15 minutes, at which point it was allowed to dry 30 seconds before being weighed. Once the sample was weighed it was squeezed until no oil could be removed from the sample, and then returned to the oil container to repeat the process. This process was repeated until the weight of the squeezed sample was equal to the weight of the un-squeezed wet sample. The kinetics-malted barley was determined by adding a 1-gram sample of the sorbent directly to $120 \mathrm{ml}$ of waste fuel. The sorbent was left in the mixture for varying times 0 to 35 minutes every 5 minutes, then removed and allowed to dry for 30 seconds before its wet weight was obtained for each sample.

\section{Results and Discussion}

\subsection{Sorption capacity}

The potato peels was examined for bouncy and the water and oil up take as shown in Figures 1 and 2. It was found that the sorbent was passed the bouncy test and the water and oil uptake showed very small amount of water was adsorbed by this sorbent which is encouraging to use it in waste fuel adsorption from the surface of water. The amount of oil that adsorbed from the sorbent was equal to $1.95 \mathrm{~g}$ oil $/ \mathrm{g}$ of sorbent while the amount of water was $0.2 \mathrm{~g}$ water/ $\mathrm{g}$ of sorbent.

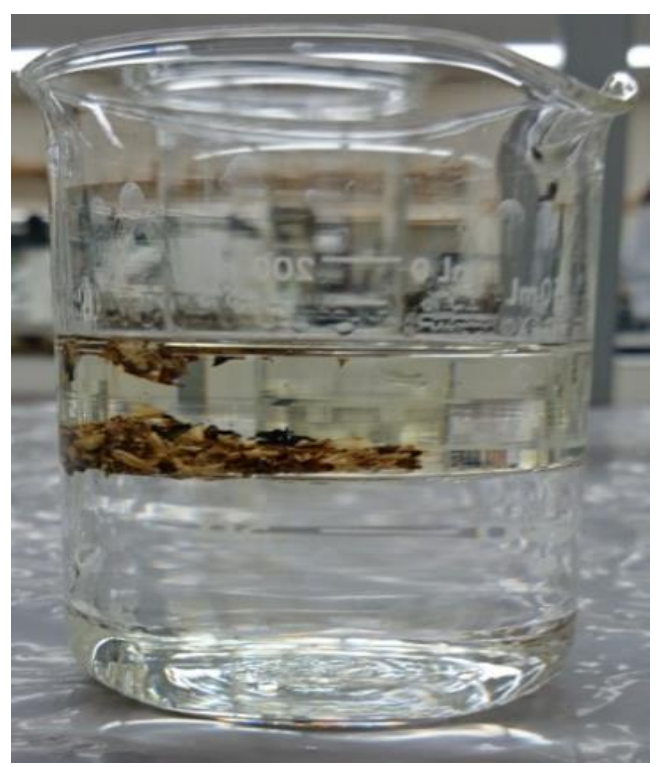

Fig. 1: Bouncy test for spent malted barley. 


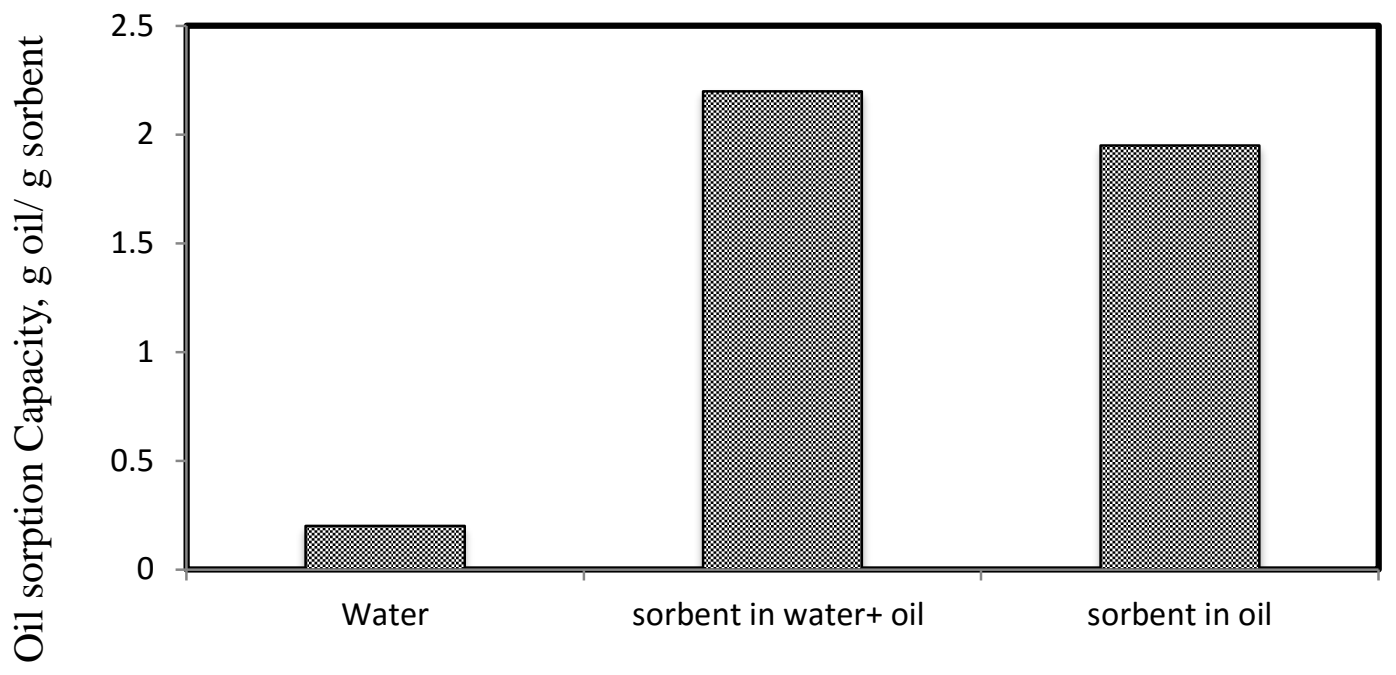

Fig. 2: Oil sorption capacity in oil, water, and oil water mixture.

The sorbent capacity was studied to determine the time required to reach equilibrium state [Figure 3]. It was shown that the equilibrium state of adsorption process was reached after 30 minutes of mixing the sorbent with the waste fuel- water mixture. This means that the short period cycle of 15 minutes is not enough to study the adsorption performance of potato peels. The average adsorption capacity was equal to $2.2 \mathrm{~g}$ of oil/g of sorbent.

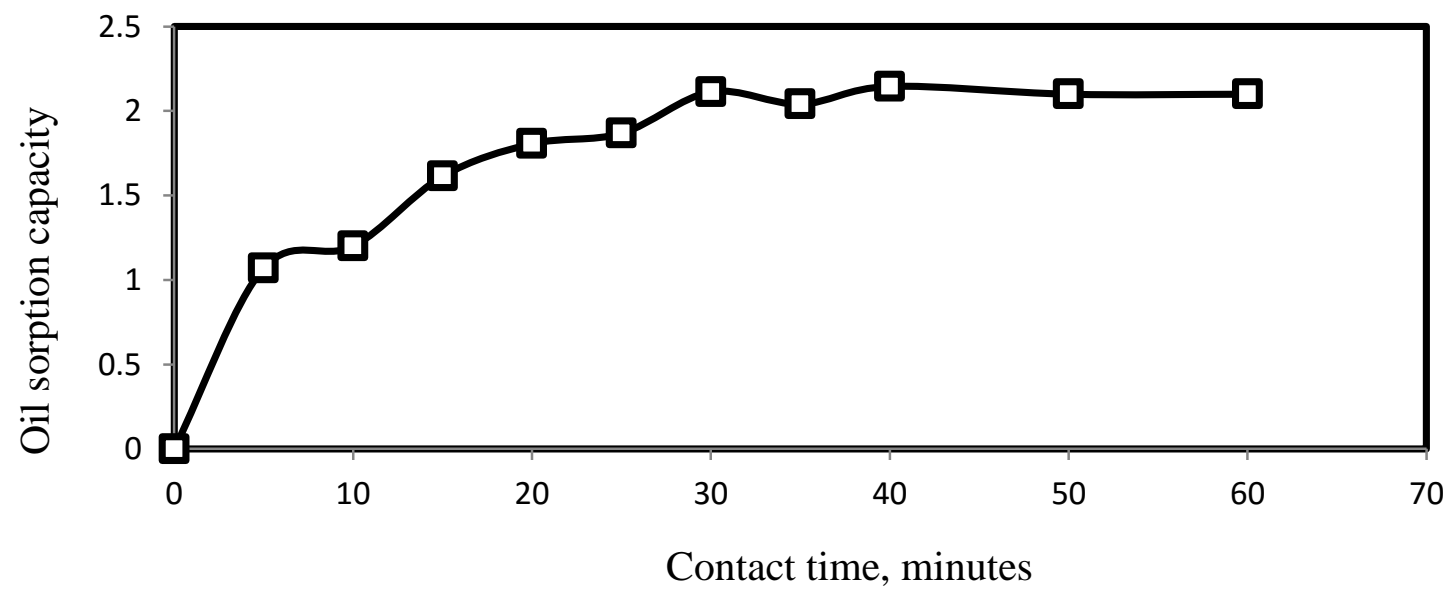

Fig. 3: Oil soprtion capacity as function of contact time.

The amount of adsorbed waste fuel by changing the amount of sorbent material is shown in Figure 4. In this figure, the amount of oil was increased with the amount of sorbent. This is in agreement with theory that the amount of adsorbed oil increased with the amount of sorbent material.

The oil sorption capacity of potato peels using batch, static state, and short period adsorption is plotted in Figure 4. According to Figure 3, the adsorption process needs about 30 minutes to reach equilibrium. So Figure 4 is extended to work as a medium period adsorption process. The oil sorption capacity is shown in Figure 5. 


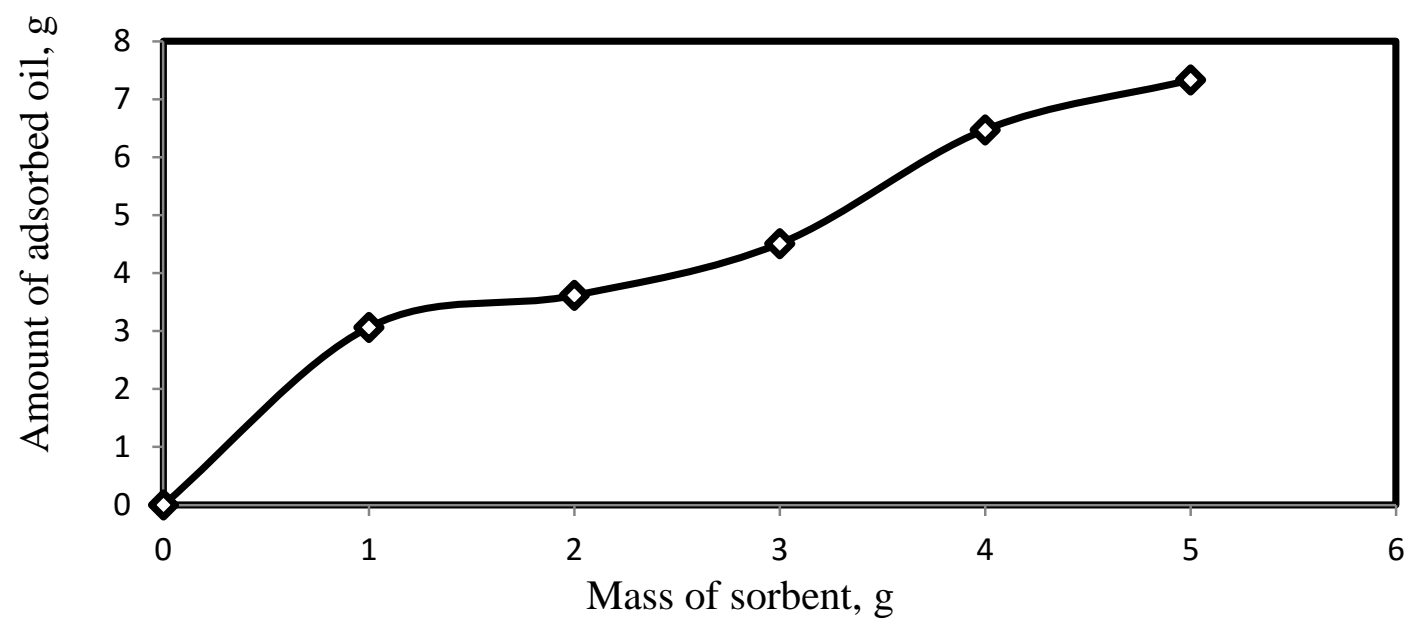

Fig. 4: Amount of waste fuel oil adsorbed from the surface of wate with amount of sorbent using short period adsorption process.



Fig. 5: Oil sorption capacity of potato peels with mass of sorbent using short period adsorption process.

The oil sorption capacity is increased from $\mathrm{g}$ oil/g sorbent to $\mathrm{g}$ oil /g sorbent. This means an increase of $\%$ in oil adsorption capacity.

\subsection{Retention time}

The retention time demonstrates how well the potato peels will hold the waste fuel oil [Figure 6]. It is an important parameter because it is related to the time of removal of sorbents from the contaminated area to assigned location. The sorbent with good retention time is needed all the times. The retention time was reduced from about $2 \mathrm{~g}$ oil / $\mathrm{g}$ sorbent to 1.232 after 1 minutes, this means that the loss of oil is about 38.4 of the original adsorption capacity and this reduced to 38.84 after 1.5 minutes of drainage. There was no significant change in waste fuel oil retained per gram of sorbent after used for the tested interval (0.5-3 minutes). The results appear to be somewhat sporadic and this time. These results are in agreement with other works $[8,9,15]$. 


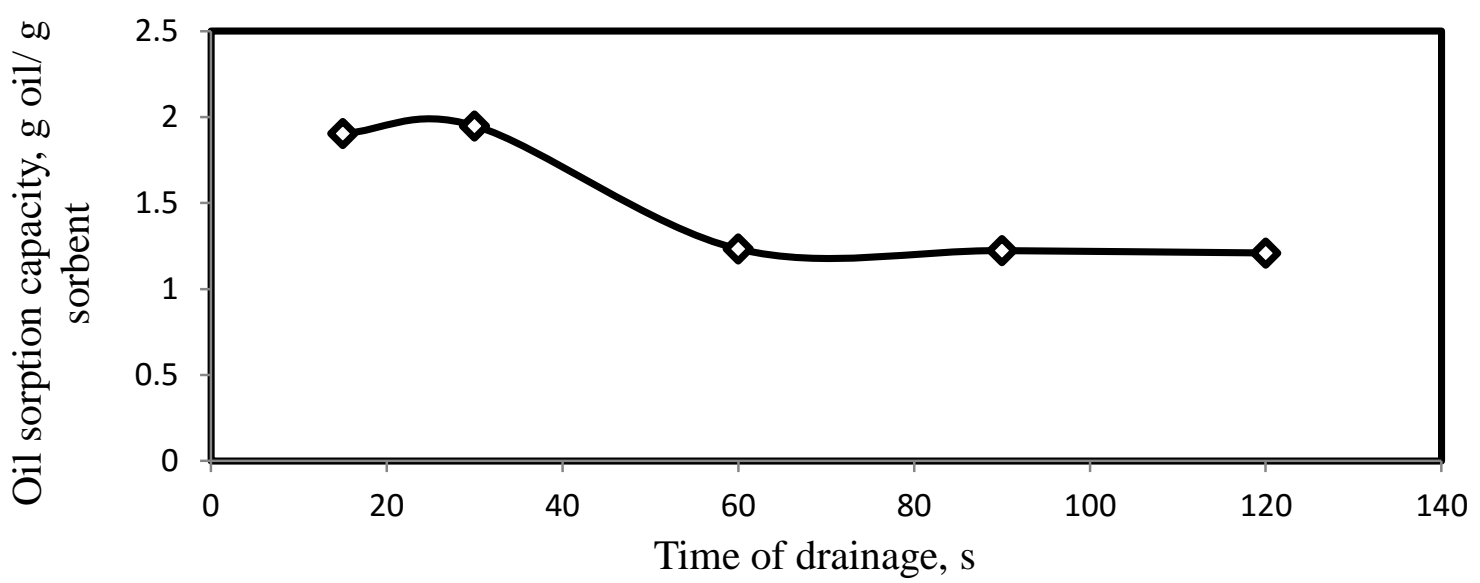

Fig. 6: Oil retention as function of adsorption capacity.

\subsection{Reusability}

When testing the potato peels powder for its reusability, the waste fuel oil was squeezed out of the sorbent by applying pressure to the saturated test sorbent. The process of squeezing the sample causes irreversible deformation of the sample and caused ultimately decreases its capacity for further adsorb of waste fuel oil. The process of squeezing the sample causes irreversible deformation of the sample and ultimately decreases its capacity to further waste fuel oil adsorption. The oil sorption capacity of potato peels powder had decreased by $65 \%$ after three time of usability. The low usability times were due to the nature of the materials that could not adsorb oil efficiently.

\subsection{Kinetic}

From Figure 3, it is clearly shown that waste-lubricating oil reached equilibrium adsorption state after 30 minutes of immersing it in water contamination with waste lubricating oil. The sorption capacity of the potato peels powder remains relatively constant at an average of $2.15 \mathrm{~g}$ waste lubricating oil / $\mathrm{g}$ sorbent. Furthermore, it should be noted that when a sample of equivalent size was briefly immersed in the sorbate, removed, and weighed it also obtained a sorbency of $2.15 \mathrm{~g} / \mathrm{g}$. This demonstrates that the effect of sorption time is quite minimal (for periods of up to and including 30 minutes). The sorption capacity of each sample may have deviated less if the particle size of each sample had been regulated and consistent - however, it was not.

In order to obtain relevant parameters, batch kinetic data in conjunction with proper models will be studied for both first and second orders adsorption kinetic models. The first order adsorption kinetics can applied using the following equation:

$$
\frac{d q}{d t}=k 1(q e-q)
$$

Where $q e$ is the equilibrium amount of adsorbed contaminant $(\mathrm{g})$ per unit mass $(\mathrm{g})$ of sorbent material, $q$ is adsorbed amount at any time $\mathrm{t}$, and $k 1$ is the first - order rate constant $(1 / \mathrm{min})$. Integrating the above equation with the limit $q=0$ at time $t=0$ gives:

$$
\ln \frac{q e-q}{q e}=-k 1 t
$$

This equation also can be written as:

$$
\ln (q e-q)=-k 1 t+\ln (q e)
$$


The plot of $\ln (q e-q)$ vs. time gives a straight line with slop equal to $-k 1$ and intercept of $\ln (q e)$.

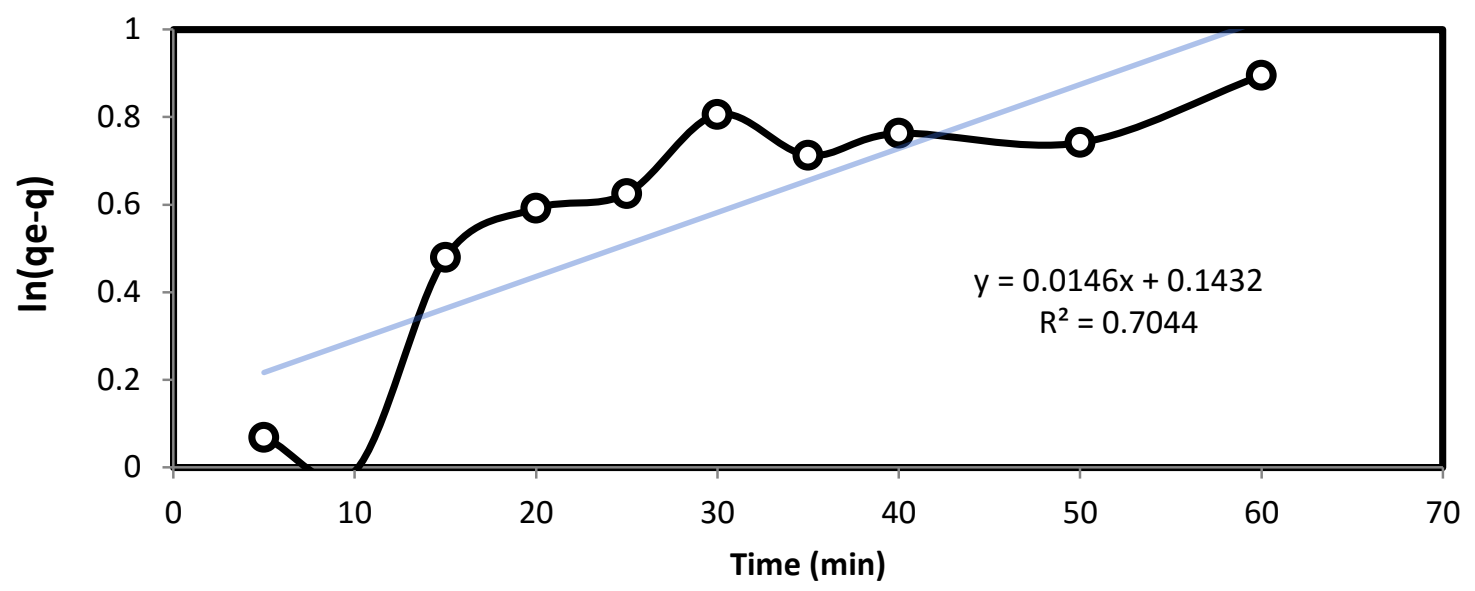

Fig. 7: First order adsorption of water remediation process.

On the other hand, the second order adsorption kinetics is described by the following equation.

$$
\frac{d q}{d t}=k 2(q e-q)^{2}
$$

$k 2$ is the second order rate constant ( $g$ sorbent per g contaminant per minute). The integration of this equation with the limit $\mathrm{q}=0$ at time $\mathrm{t}=0$ gives:

$$
\left\{\frac{1}{q e-q}\right\}-\left\{\frac{1}{q e}\right\}=-k 2 t
$$

Or

$$
\left\{\frac{t}{q}\right\}=\left\{\frac{t}{q e}\right\}+\left\{\frac{1}{k 2 \mathrm{qe}^{2}}\right\}
$$

The plot of $\left\{\frac{t}{q}\right\}$ vs. time gives a straight line with the slope $\left\{\frac{1}{q e}\right\}$ and intercept of $\left\{\frac{1}{k 2 \mathrm{qe}^{2}}\right\}$. The experimental data of adsorbing oil from the surface of water using spent malted barley fits the second order kinetics as shown in Figure 7 . The kinetic parameters such as the rate constant and qe are computed from the slope and the intercepts of the fitted curve.



Fig. 7: The second order plot of adsorption process of waste fuel oil from the surface of water using potato peels powder. 
The kinetic parameters such as the rate constant and qe are computed from the slope and the intercepts of the fitted curves and reported in Table (2).

Table 2: Summary of kinetic parameters for WTCF sorption.

\begin{tabular}{|l|l|l|l|l|l|l|}
\hline Sorbent & Kinetic & Slope & Intercept & $\mathrm{R}^{2}$ & qe & $\begin{array}{l}\text { Rate } \\
\text { constant }\end{array}$ \\
\hline \multirow{2}{*}{$\begin{array}{l}\text { Potato } \\
\text { peels }\end{array}$} & First order & 0.0146 & 0.1432 & 0.7044 & 1.1540 & -0.0146 \\
\cline { 2 - 7 } & Second order & 0.1771 & 2.2181 & 0.9558 & 0.4508 & 2.2185 \\
\hline
\end{tabular}

From the values of the slope, qe and $\mathrm{R}^{2}$, it is quite accepted to say that the second order kinetics is better fit for potato peels powder for waste lubricating oil removal from oil spill to the water surface.

\section{Conclusion}

Potato peels powder was used as sorbent material for adsorbing waste lubricating oil from the surface of seawater. This waste biomass material is available in huge quantities in Canada from all the fast food restaurants and it can be used as sorbent material after careful treatment. The evaluation of potato peels powder showed that it can adsorb oil up to more than $2.15 \mathrm{~g}$ of oil /g of sorbent and it can adsorb oil in a very fast process and can keep the oil for long time. This is a very important property which called oil retention. The adsorption equilibrium isotherm showed that the adsorption process is fitted to second order adsorption process.

\section{Acknowledgment}

The authors would like to thank the University of Regina for providing all facilities to achieve this work.

\section{References}

[1] T. Paulauskienè, I. Jucikè, N. Juščenko, and D. Baziukè, "The Use of Natural Sorbents for Spilled Crude Oil and Diesel Cleanup from the Water Surface,” Water, Air \& Soil Pollution, vol. 225, no. 6, pp. 1, 2004.

[2] L. Teik-Thye and X. Huang, "Evaluation of hydrophobicity/oleophilicity of kapok and its performance in oily water filtration: Comparison of raw and solvent-treated fibers," Industrial Crops and Products, vol. 26, no. 2, pp. 125-134, 2007.

[3] A. Bazargan, J. Tan, C. W. Hui, and G. McKay, "Utilization of rice husks for the production of oil sorbent materials," Cellulose, vol. 21, no. 3, pp. 1679-1688, 2014.

[4] O. A. Wahab, "Assessment of raw luffa as a natural hollow oleophilic fibrous sorbent for oil spill cleanup," Alexandria Engineering Journal, vol. 53, no. 1, pp. 213-218, 2014.

[5] M. A. Abdullah, A. Ur Rahmah, and Z. Man, "Physicochemical and sorption characteristics of Malaysian Ceiba pentandra (L.) Gaertn as natural oil sorbent," Journal of Hazardous Materials, vol. 177, pp. 683-691, 2010.

[6] M. Jansi Rani, M. Murugan, P. Subramaniam, and E. Subramanian, "A study on water hyacinth Eichhornia crassipes as oil sorbent," Journal of Applied and Natural Science, vol. 6, no. 1, pp. 134-138, 2014.

[7] I. A. H. Al Zubaidy, U. Zaffar, N. Chowdhury, N. Mustafa, V. Varughese, R. Ahmed, R. A. Alharmoudi, A. Shahid, and E. E. Gomes, "Adsorption study of bio-degradable natural sorbents for remediation of water from crude oil," in International Conference on Natural Science and Environment, 2014.

[8] I. A. Al Zubaidy, "Effect of activation of date palm kernel powder on the remediation process of oil polluted water," in International Journal of Environmental Pollution and Remediation, vol. 1, no. 1, 2012, pp. 54-59.

[9] P. Tontiwachwuthikul, I. Al Zubaidi, J. Walsh, J. Szarkowicz, J. Humeny, and J. Fedorchuk, "Kinetic study of the remediation of water from diesel oil spill using spent malted barley," in $65^{\text {th }}$ Canadian Chemical Engineering Conference, 2015.

[10] M. Husseien, A. A. Amer, A. El-Maghraby, and N. A. Taha, "Experimental investigation of thermal modification influence on sorption qualities of Barley Straw," Journal of Applied Sciences Research, vol. 4, no. 6, pp. 652-657, 2008. 
[11] A. G. El-Said, N. A. Badawy, and A. Abd El Pasir, "Comparison of Synthetic and Natural Adsorbent for Sorption of Ni (II) Ions from Aqueous Solution," Nature and Science, vol. 8, no. 11, pp. 86-94, 2010.

[12] A. Maleki, H. Daraei, F. Khodaei, K. Bayazid-Aghdam, R. Rezaee, and A. Naghizadeh, "Investigation of potato peelbased bio-sorbent efficiency in reactive dye removal: Artificial neural network modeling and genetic algorithms optimization," J. Adv. Environ. Health Res., vol. 1, no. 1, pp. 21-28, 2013.

[13] H. A. Omar and M. S. Sayed, "Potential of Potato Periderm and Potato Periderm Ash for the Removal of Co+2 and Eu+3 Ions from Liquid Radioactive Waste Solutions," in International Journal of Environmental Engineering Science, vol. 2, no. 2, pp. 179-192, 2011.

[14] Potato peels in Five Guy restaurants. [Online]. Available at: http://www.isu.edu/magazine/fall11/fry-guy.shtml

[15] N. Yodsanti, "Recovery, analysis, and utilization of potato peels," MSc thesis, University of Alberta, 2002.

[16] M. Husseien, A. A., Amer, A. El-Maghraby, N. A. Taha., "Availability of barley straw application on oil spill cleanup," Int. J. Environ. Sci. Tech., vol. 6, pp. 123-130, 2009. 